\title{
Engaging frontline health providers in improving the quality of health care using facility-based improvement collaboratives in Afghanistan: case study
}

Mirwais Rahimzai ${ }^{1}$, Ahmad Jan Naeem ${ }^{2}$, Silvia Holschneider ${ }^{3^{*}}$ and Ahmad Kamran Hekmati ${ }^{4}$

\begin{abstract}
Background: Access to health services in Afghanistan has expanded in the last decade; however, gaps in care quality and outcomes of care remain a challenge. Recognizing these gaps, in 2009 the USAID Health Care Improvement Project $(\mathrm{HCl})$ began assisting the Ministry of Public Health $(\mathrm{MoPH})$ in Afghanistan to improve the quality of health services. Though eventually scaled up to nine provinces, in 2009 the $\mathrm{MoPH}$ and $\mathrm{HCl}$ began developing and testing a facility-based, scalable model of maternal and newborn interventions in health facilities in Kunduz and Balkh provinces and in several large public and private hospitals in Kabul.
\end{abstract}

Case description: To address these issues, the $\mathrm{MoPH}$ and $\mathrm{HCl}$ applied the collaborative improvement approach, which links the efforts of multiple teams focused on making improvements in the same area of care to achieve the same aims. As demonstration sites, two provincial level quality improvement teams (QITs) were established in Kunduz and Balkh provinces that, in turn, supported 25 facility-level QITs chosen by purposive sampling. In addition, three government maternity hospitals and three private hospitals in Kabul were selected by the MoPH to participate in a demonstration hospital collaborative. Measurable gains were achieved in these demonstration sites for use of the partograph, compliance with antenatal care counseling, vaginal births for which all three elements of active management of the third stage of labor were performed, and compliance with newborn and postnatal care standards.

Discussion and evaluation: Quality of care can be significantly improved by engaging teams of frontline workers to identify problems and find local solutions for those problems. Based on the results achieved in Kunduz, Balkh, and Kabul, the collaborative improvement work was expanded from 2010-2012 to seven more provinces. The results achieved on the ground also led the MoPH to establish a unit for quality and a national health care quality improvement strategy for Afghanistan.

Conclusions: Afghanistan demonstrates that even in fragile states, measurable improvements in actual patient care at the frontlines of service delivery can be achieved while systematically building capacity at all levels of the health system through national leadership and policy making.

Keywords: Collaborative improvement, Maternal and newborn health, Quality improvement, Quality improvement teams

\footnotetext{
* Correspondence: sholschneider@urc-chs.com

${ }^{3}$ University Research Co., LLC (URC), 7200 Wisconsin Ave., Suite 600,

Bethesda, MD 20814-4811, USA

Full list of author information is available at the end of the article
} 


\section{Background}

The Ministry of Public Health (MoPH) of the Government of the Islamic Republic of Afghanistan has made strides since it began to rebuild the Afghan public health system in 2002 [1]. With the support of donors and contracting out through nongovernmental organizations (NGOs), a Basic Package of Health Services (BPHS) and Essential Package of Hospital Services (EPHS) were developed to form the core of service delivery in health care facilities and hospitals [2]. As a result, many health indicators greatly improved, and access to health services increased [3]. In 2004, multiple donor programs and the $\mathrm{MoPH}$ began a series of initiatives to improve health care quality, with some success, but fragmentation, poor coordination, and sustainability remained as issues to be addressed [4]. Despite these achievements in the health sector, Afghanistan still lagged behind countries in the region in health outcomes, particularly in the areas of maternal, infant, and child health.

Recognizing these gaps, in 2004 the MoPH began a series of initiatives to improve health care quality, increasing the number of partners working on health care in the country. One such initiative began in 2008, when the United States Agency for International Development (USAID) Mission in Afghanistan requested that the USAID Health Care Improvement Project (HCI) initiate assistance to the $\mathrm{MoPH}$ and implementing partners to help them improve the quality of health services nationwide. Specifically, HCI was asked to focus on improving maternal and child health in Balkh and Kunduz provinces as the initial locations for HCI assistance. At the time, neither of these provinces received other USAIDsupported assistance. In these provinces, HCI would test a facility- and community-based scalable model of antenatal, delivery, and postpartum care interventions.

Based on extensive experience assisting other countries to improve obstetric and newborn care, $\mathrm{HCI}$ proposed the application of the collaborative improvement approach to "frontline" health providers. This approach links the efforts of multiple teams focused on making improvements in the same area of care to achieve the same aims, as the improvement strategy in Afghanistan. Collaborative improvement is a structured improvement approach that organizes a large number of teams or sites to work together for a 12- to 24-month period to achieve significant improvements in a specific area of care. $\mathrm{HCI}$ and its predecessor, the USAID Quality Improvement Project (QAP), adapted the Breakthrough Series improvement collaborative model (developed by the Institute for Healthcare Improvement) and have supported over 100 collaboratives in some 20 low- and middle-income countries since 2003, producing excellent results in a wide variety of care areas. Data from developing country settings show that the collaborative improvement approach can produce significant, sustained gains in compliance with standards and in outcomes in maternal and child health and other health areas [5].

The collaborative improvement approach combines traditional quality improvement methods of team work, process analysis, introduction of standards, measurement of quality indicators, training, job aids, and coaching with techniques based on social learning and diffusion of innovation theories. In a collaborative, teams of health care providers work independently to test changes in how to improve the delivery of care. Teams use a common set of indicators to measure the quality of the care processes the collaborative is trying to improve and, where possible, the desired health outcomes. The collaborative organizes regular sharing of results among teams through learning sessions in which teams learn from each other about which changes were successful and which were not. This results in a dynamic improvement strategy in which many teams working on related problem areas can learn from each other in a way that facilitates rapid dissemination of successful practices. In its emphasis on spread and scale-up of improvements, the improvement collaborative model offers a powerful tool in the arsenal of proven improvement methods [6].

Improvement collaboratives were designed by $\mathrm{HCI}$ and Afghanistan-based experts to introduce high-impact, evidence-based interventions in maternal and newborn care at both the facility and community levels. As shown in Figure 1, the strategy envisioned linking the efforts of quality improvement teams (QITs) at the regional hospital (RH), district hospital (DH), comprehensive health center $(\mathrm{CHC})$, basic health center $(\mathrm{BHC})$, health post $(\mathrm{HP})$, and community levels to first demonstrate results and then scale up the changes introduced at each level to improve

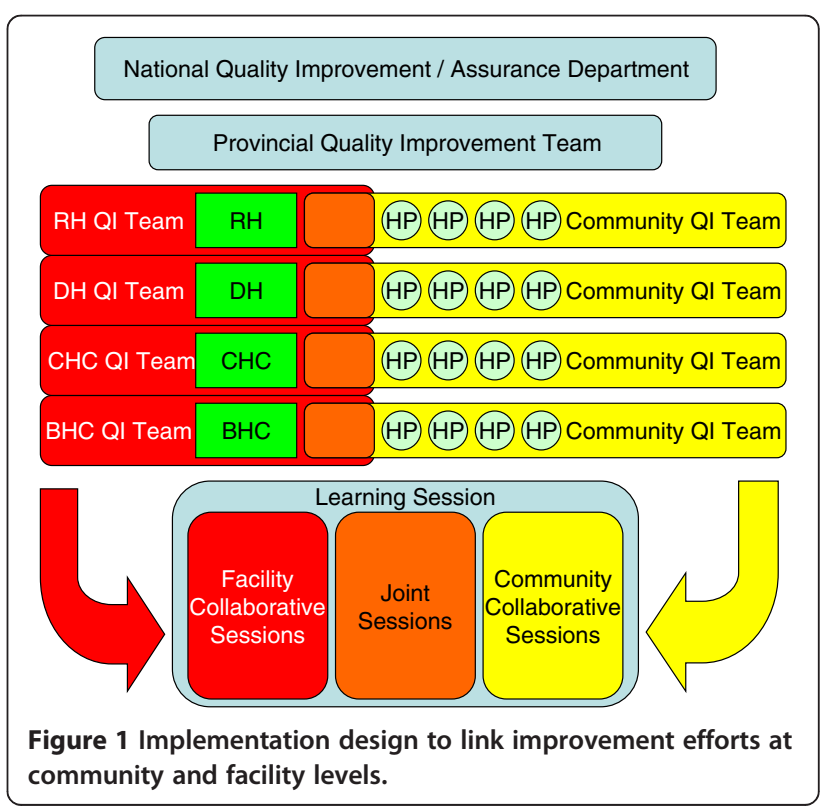


care to other facilities in the province. The purpose of the facility-level collaborative improvement was to focus on improving antenatal care (ANC), routine delivery care, active management of the third stage of labor (AMTSL), postnatal care (PNC), and essential newborn care. The maternal and newborn care community collaborative would address the quality of services delivered by health posts and community health workers living in rural areas.

In parallel to the provincial level improvement work in the two northern provinces, the MoPH, USAID, and HCI decided to develop a Kabul-based hospital improvement activity as well, in part to create opportunities for senior MoPH officials to gain first-hand experience with the improvement work. In close collaboration with the MoPH, the U.S. Centers for Disease Control and Prevention (CDC), and UNICEF, HCI designed an improvement collaborative for maternity hospitals that would focus on improving delivery care, provision of AMTSL, and other high-impact interventions in three public and three private hospitals in Kabul were also selected to participate. HCI's role in the collaborative was to provide technical assistance in improvement methods, build capacity for improvement among the partners, and support the collaborative's roll-out of interventions to improve maternal and perinatal outcomes.

This article provides a case description of the facilitylevel health care improvement interventions and outcomes in Balkh and Kunduz provinces and in maternity hospitals in Kabul. While community-level improvement interventions were also carried out in Balkh and Kunduz provinces, they are not described in this article.

\section{Case description}

Development of the improvement collaboratives

In May 2009, the MoPH and HCI began the Maternal and Newborn Health Facility Demonstration Improvement Collaborative. The overall objective of this facilitybased collaborative in Balkh and Kunduz provinces was to reduce maternal and newborn mortality and morbidity through improved quality of care in basic public health facilities, including provincial hospitals, health centers, and health posts.

Balkh and Kunduz provinces were selected because at the time these provinces received no other USAIDsupported assistance; this selection would minimize overlap with existing USAID-funded technical support. The original plan was to start with $10-20 \%$ of health facilities in the two provinces and then expand within each province and to other provinces. This plan was modified because the MoPH requested earlier expansion to new provinces after seeing early positive results from the demonstration facilities in Balkh and Kunduz.

Before starting the improvement activities, HCI conducted meetings with the $\mathrm{MoPH}$ and Provincial Public
Health Departments (PPHDs) in Balkh and Kunduz to receive their buy-in to start the collaborative and to select the sites that would participate. HCI staff were hired at the provincial level and oriented on improvement methodology.

In May 2009, HCI led two orientation sessions for facilities participating in the collaborative - formally called the Maternal and Newborn Health Facility Demonstration Improvement Collaborative - to introduce the project, improvement methods, and the collaborative improvement approach. Ten to fifteen percent of the health facilities in Kunduz and Balkh provinces were selected in the demonstration phase by purposive sampling, based on their willingness to participate, recommendation by the Government, and availability of minimum, basic infrastructure to provide services.

In July 2009, health facility staff in collaboration with HCI conducted a baseline assessment of health facilities in both provinces to assess the quality of antenatal, postpartum, and newborn care and provider counseling in antenatal and postnatal care as well as the availability of essential medications and materials. HCI trained a team of HCI assessors and personnel from the provincial public health office to conduct the baseline assessment. Baseline data for the previous six months were collected from the health facility records for most of the indicators. However, there were a few indicators for which data could not be found in facility records; hence, random sampling of patients was used to collect baseline data.

Figure 2, which presents baseline data on average compliance with essential newborn care standards, shows the low level of compliance (20-60\%) with standards that the collaborative sought to improve.

HCI worked with the provincial public health authorities to establish one provincial level QIT in both provinces that would, in turn, support facility-level QITs made up of staff responsible for maternal and newborn care. The provincial QITs included Provincial Public Health Officers, representatives of BPHS- and/or EPHSimplementing NGOs, and HCI staff. Based on the baseline data, the provincial QITs prioritized a package of high-impact interventions that would be easiest to introduce, since HCI's experience had shown that collaborative improvement efforts are often more effective in the long run when they are tasked with improving intervention packages in sequence, rather than seeking to simultaneously improve multiple complex processes. The initial focus of the collaborative improvement efforts was determined to be ANC, delivery care, and immediate PNC, AMTSL, essential newborn care, tetanus vaccination for pregnant women, institutional deliveries, partograph usage, and postnatal monitoring. It was expected that a second "phase of work" (referred to as 


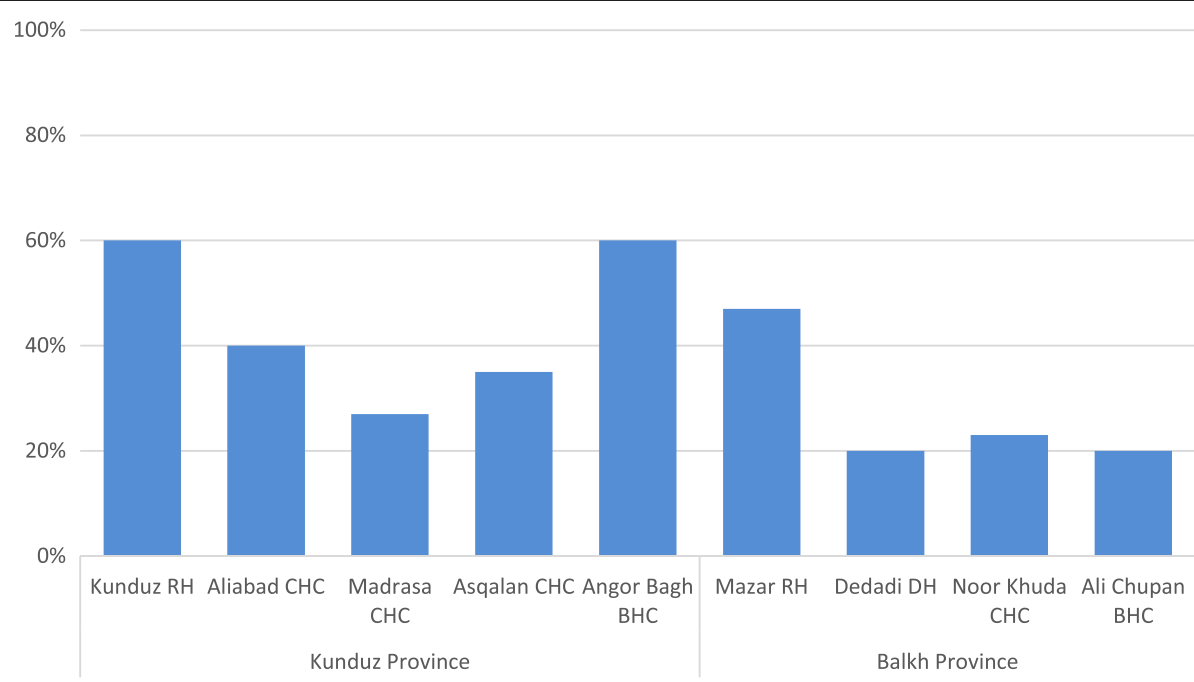

Figure 2 Baseline facility assessment findings for essential newborn care, Kunduz and Balkh provinces (July 2009).

"wave") in each province would focus on improving the prevention and case management of maternal complications, especially pre-eclampsia and eclampsia, and then be followed by a third phase of content related to preventing and treating maternal and newborn sepsis.

In Kunduz province, one regional hospital, four comprehensive health centers, eight basic health centers, and two subcenters, comprising 15 facilities in all, were selected to participate in the collaborative improvement work (Table 1). The total estimated catchment population of these facilities (or "sites") was 477,677 out of the total provincial population of 882,900 .

In Balkh province, 10 facilities - one regional hospital, one district hospital, one comprehensive health center, six basic health centers, and one subcenter - were selected to participate (Table 1). The total estimated catchment population for these 10 facilities was 533,518 out of the total provincial population of 1,144,800.

In February 2010, the MoPH and HCI also began to develop the hospital maternity care collaborative for several large public and private hospitals in Kabul, to improve the prevention and treatment of the major causes of direct maternal and neonatal mortality. Before starting the hospital collaborative, HCI conducted a series of meetings with the MoPH and hospital directors in Kabul to receive their buy-in for the collaborative approach and to prioritize interventions. Three government maternity hospitals (Malalai Maternity, Isteqlal, and Khair Khana) and three private hospitals (Shino Zada, Afghan, and Mahdi) in Kabul were selected by the MoPH to participate in the hospital collaborative (Table 1). The government hospitals were selected by the $\mathrm{MoPH}$ to participate in the collaborative because they were the biggest referral centers. The private facilities were selected by the MoPH according to the following criteria: providing delivery services, willingness to participate, and having basic infrastructure. At the time, the estimated catchment population for these facilities, which attend most deliveries in the city, was $3,449,800$ out of approximately 4,000,000 residents of Kabul.

\section{The role of quality improvement teams}

For Balkh and Kunduz provinces, HCI, in collaboration with the PPHDs, held the first provincial learning sessions in October 2009 for the QITs. Each facility had approximately one QIT, and each team comprised permanent as well as need-based members. Permanent members were facility-level OB/GYN doctors and/or midwives (approximately two per facility); administrative staff and sometimes management of the hospital were

Table 1 Numbers and types of health facilities participating per province (2009-2013)

\begin{tabular}{|c|c|c|c|c|}
\hline Wave & Province & Dates & \# of facilities participating & Type of facilities \\
\hline \multirow[t]{3}{*}{ Demonstration } & Balkh Initial & $4 / 2009-12 / 2012$ & 10 & $\begin{array}{l}1 \text { regional hospital; } 1 \text { district hospital; } 1 \text { comprehensive } \\
\text { health center; } 6 \text { basic health centers; } 1 \text { subcenter }\end{array}$ \\
\hline & Kunduz & $4 / 2009-1 / 2012$ & 15 & $\begin{array}{l}1 \text { regional hospital; } 4 \text { comprehensive health centers; } 8 \text { basic } \\
\text { health centers; } 2 \text { sub-centers }\end{array}$ \\
\hline & Kabul & $4 / 2010-3 / 2013$ & 6 & 3 government maternity hospitals; 3 private hospitals \\
\hline
\end{tabular}


involved on a need-based basis. In total 40-50 representatives from each province were invited. Most of the providers selected were females, as they were working in labor and delivery. These female providers were highly motivated by the opportunity to learn from their peers working in similar settings. At each provincial learning session, health facility QITs were introduced to the results of the baseline assessments of the indicators related to the quality of maternal and newborn health care services. In addition, they were introduced to interventions related to improving ANC counseling, birth preparedness, hand hygiene, AMTSL, and essential newborn care. Each team left the learning session with a draft action plan of aims and the changes they would discuss for implementation in their facilities after the session.

Following the first learning session, two rounds of coaching visits were performed to all targeted health facilities in both provinces by $\mathrm{HCI}$ and PPHD staff. The purpose of such coaching in health care improvement work is to provide technical and moral support to QITs to improve their performance by: ensuring that QIT members have a positive attitude toward quality improvement; working with the QITs to master the principles of QI; strengthening technical and analytical skills; strengthening capacities for planning, implementation, and follow-up for corrective action plans; developing personal and professional skills of individuals and teams; and encouraging positive changes in performance through taking initiative, internalizing the institution's goals and values, continued learning, sustaining a high level of performance, and creating and maintaining positive working relationships and maintaining respect [7]. During these coaching visits, facility QITs were supported to introduce and test change ideas that had been discussed in the learning sessions and review their data on the QI indicators that had been defined with provincial health teams in order to measure the overall results of the improvement work.

For the Kabul hospital maternity care collaborative in December 2009, HCI conducted a two-day orientation workshop for the hospital directors and team members to discuss QI methodology and the high-impact interventions that would be the focus of the hospital collaborative. QITs in Kabul hospitals were established in the same way as in facilities in other provinces. These teams had permanent as well as need-based members. Permanent members were those who were working on the process to be improved within delivery and newborn wards. Administrative staff and sometimes hospital management staff were involved as needed. In order to develop a comprehensive intervention package and measures for monitoring, a one-day session was convened in Kabul in February 2010, where representatives of all the participating hospitals and MoPH officials participated. Priority high-impact intervention areas that were decided upon and that were the focus of the Kabul Maternity Hospital Collaborative are listed below.

1. Improve management of leading causes of maternal and neonatal mortality - improved complications care (e.g., focus on improved detection and case management of pre/eclampsia, maternal and newborn sepsis, and newborn asphyxia)

2. Improve hospital childbirth care processes by care phase

a. Initial triage: re-organization of care processes for improved detection of risks and complications requiring immediate action to ensure compliance with national hospital standards and minimize "third delay" once woman arrives at hospital

b. Labor care: use of partogram for early detection of obstructed labor, regular monitoring of cervical dilation and fetal descent, regular monitoring of fetal heart rate, and regular monitoring of maternal temperature and blood pressure

c. Vaginal delivery care/immediate postpartum care: re-organization of care to ensure compliance with standards

d. Postpartum and discharge care: Re-organize care to promote routine, high-impact postpartum interventions (i.e., exclusive breastfeeding, prevention of newborn hypothermia, low birth weight infants, kangaroo care); regular surveillance for hemorrhage, maternal or newborn sepsis, special care of low birth weight infants; family planning; pre-discharge counseling (danger signs, follow up, etc.)

3. Improve routine, high-impact childbirth care: Improve compliance with routine, high-impact interventions during intrapartum period: infection prevention (hand washing, instrument decontamination, high-level sterilization), AMTSL, essential newborn care, family planning, etc.

4. Improve referrals

The first learning session of the Kabul maternity hospital collaborative took place in April 2010. The learning session was mainly focused on orienting participants on quality improvement methodology, selection of improvement priorities, and identification of QITs in facilities. In May 2010, the six hospitals completed their baseline assessments, focusing on maternal and newborn outcomes, complications management, maternal and newborn postnatal care, and medical records. QITs were established in all six hospitals and visited regularly by HCI staff, who provided on-the-job training on the correct use of the partograph, early detection of postpartum hemorrhage, AMTSL, and essential newborn care. Health facilities were supported in implementing change ideas as well as 
collecting, compiling, and plotting their data on time series charts and analyzing their performance and outcomes.

\section{An example of the work of one QIT in Balkh province}

In Balkh province, for example, a QIT was established in a regional hospital serving five nearby provinces and approximately 5 million people. The facility QIT reviewed available data from hospital records on maternal mortality and decided to improve the use of the partograph for every woman delivering in the hospital. While the data showed that in $67 \%$ of deliveries a partograph was used, a closer look at the partograph records by the QIT members revealed that in only $13 \%$ of the cases, the partograph was completed properly. The QIT met and brainstormed on the causes of not properly filling out a partograph or not filling it out correctly. A number of issues was identified, including: 1) there was no regular supply of partograph paper, and sometimes the paper was not being supplied for six months; 2) there was no common definition of a completed partograph, and sometimes when only the name of the patient was entered, it was considered a completed partograph; 3) a mechanism to ensure the use of the partograph for decision making during labor within the hospital did not exist; and 4) there were some new health workers who did not know how to use a partograph. Subsequently, the QIT came up with a number of change ideas that they wanted to test one by one. The initial change was to train every birth attendant on how to fill out a partograph. This training was conducted by a trainer who was already working in the hospital. Data were collected for two weeks to see improvement in the use of the partograph. However, the data showed that improvement in partograph use was insignificant after the training. In the meantime the QIT members worked on a common definition of what comprised completed partographs, which was communicated to everyone during the training. The next change that was introduced was a review of completed partographs during the "morning reports" (i.e., regular morning meetings by staff), where 10 out of all the completed partographs would be sampled and then reviewed by the facility QIT. This change significantly improved use of partographs. The QIT members discussed the issue of the irregular supply of partograph paper with the director of the hospital and provided specific suggestions to improve its supply. Administrative staff of the hospital would order partograph papers for six months and stock them in the hospital. They would review their stock every three months and make sure that they had stocked a supply for the next six months. Data showed that with these simple changes the hospital never ran out of partograph papers for the next two years.
Establishing these changes did not come without challenges: Human nature is often resistant to change, sometimes with valid reasons for the resistance. If health workers were told to change their behavior in a day or a week, it would be very difficult to make happen. Also, sometimes health workers are expected to solve problems that are beyond their ability to solve. In the case study, health workers were supposed to use a partograph for each delivery; however, there was limited availability of partograph sheets. Similarly, other aims that the teams wanted to achieve in ANC were not possible due to lack of equipment or non-functional state of equipment. This created fatigue and a sense of helplessness. QITs engaged these workers in identifying local problems and classifying them in to those that were solvable within the facility and those that could be solved only with the support of district and provincial officials, communicating and following up on them.

\section{Spreading the improvement work}

Within six months of the start-up of the collaboratives in Kunduz and Balkh provinces, facility QI teams were showing strong gains in a number of quality indicators, particularly second-dose tetanus toxoid vaccination of pregnant women, ANC counseling with key messages, delivery of essential newborn care, and use of the partograph. The $\mathrm{MoPH}$ was keenly interested in spreading the maternal and newborn interventions to additional provinces. Based on the results achieved in Kunduz and Balkh, the facility and community maternal and newborn health collaborative improvement work was expanded over time in two waves to seven more provinces. New provinces and facilities were selected by the MoPH. High-impact interventions were introduced in phases, with each phase lasting about a year. As soon as a "change package" (a set of proven effective changes) was identified, it was gradually expanded to other health facilities within the provinces and then to new provinces in consultation with the MoPH. Expansion was planned through gradual inclusion of new health facilities in each collaborative, and through engagement of Government officers from the provincial public health office who could then serve as change agents within the system to introduce the change package. In late 2010, maternal and newborn health collaborative improvement activities were initiated in Bamyan, Herat, and Parwan provinces (Wave 1). In 2011 and 2012, improvement activities were initiated in Wardak (October 2011), Samangan (November 2011), Saripul (November 2011), and Logar (April 2012) provinces (Wave 2).

By the end of 2012, the maternal and newborn health facility collaborative improvement interventions had reached 85 health facilities in the nine HCI-targeted provinces, achieving measurable gains in quality of maternal 
and newborn care for an estimated total catchment population of 1,586,084 (about 24\% of the total population of the nine provinces). The largest gains achieved by the sites included:

- Use of the partograph, which averaged 92.0\% during the last three months that data were collected, up from baseline levels of around 20\% (Figure 3).

- Compliance with antenatal care counseling standards, which averaged $91.5 \%$ during the last three months of data collection, up from baseline levels of under $40 \%$.

- Compliance with postnatal care standards, which averaged $85.1 \%$ during the last three months of data collection, up from baseline levels of under $30 \%$.

- Percentage of vaginal births in which all three elements of AMTSL were performed (i.e., oxytocin administered within one minute of delivery, controlled cord traction, and uterine massage), which averaged $87.3 \%$ during the last three months of data collection, up from baseline levels of under $50 \%$ in Balkh and Kunduz and below $10 \%$ in the other provinces (Figure 4).

- Compliance with essential newborn care standards (i.e., drying and wrapping the newborn, umbilical cord care, and immediate breastfeeding), which averaged $90.3 \%$ during the last three months of data collection, up from baseline levels of around 50\% in Balkh and Kunduz and below 10\% in the other provinces (Figure 5).
- The percentage of births in which the newborn was put to breast within the first hour after birth increased from less than $25 \%$ at baseline to $90.7 \%$ in the last three months of data collection in 2012 (Figure 6).

- Percentage of mothers who are able to cite at least two maternal and newborn danger signs after delivery increased from less than $40 \%$ at baseline to $88.2 \%$ in the last three months of data collection (Figure 7).

During the 48 separate learning sessions conducted by $\mathrm{HCI}$ in the nine provinces, 1,175 health workers were trained in evidence-based maternal and newborn care practices and quality improvement methods. In FY13, the USAID Mission in Afghanistan directed HCI to complete provincial level support by December 2012. Support for quality improvement (QI) activities at the health facility level was transferred to provincial authorities of the MoPH and NGO implementing partners.

QITs were taken over by the MoPH through implementing organizations in provinces and by the public hospitals in Kabul. Most of these QI teams are continuing to improve care with the support of the Unit for Improving Quality in Healthcare of the MoPH, which was established with the support of $\mathrm{HCI}$ and was adopted by the MoPH in to its administrative structure (see "Discussion and evaluation" section).

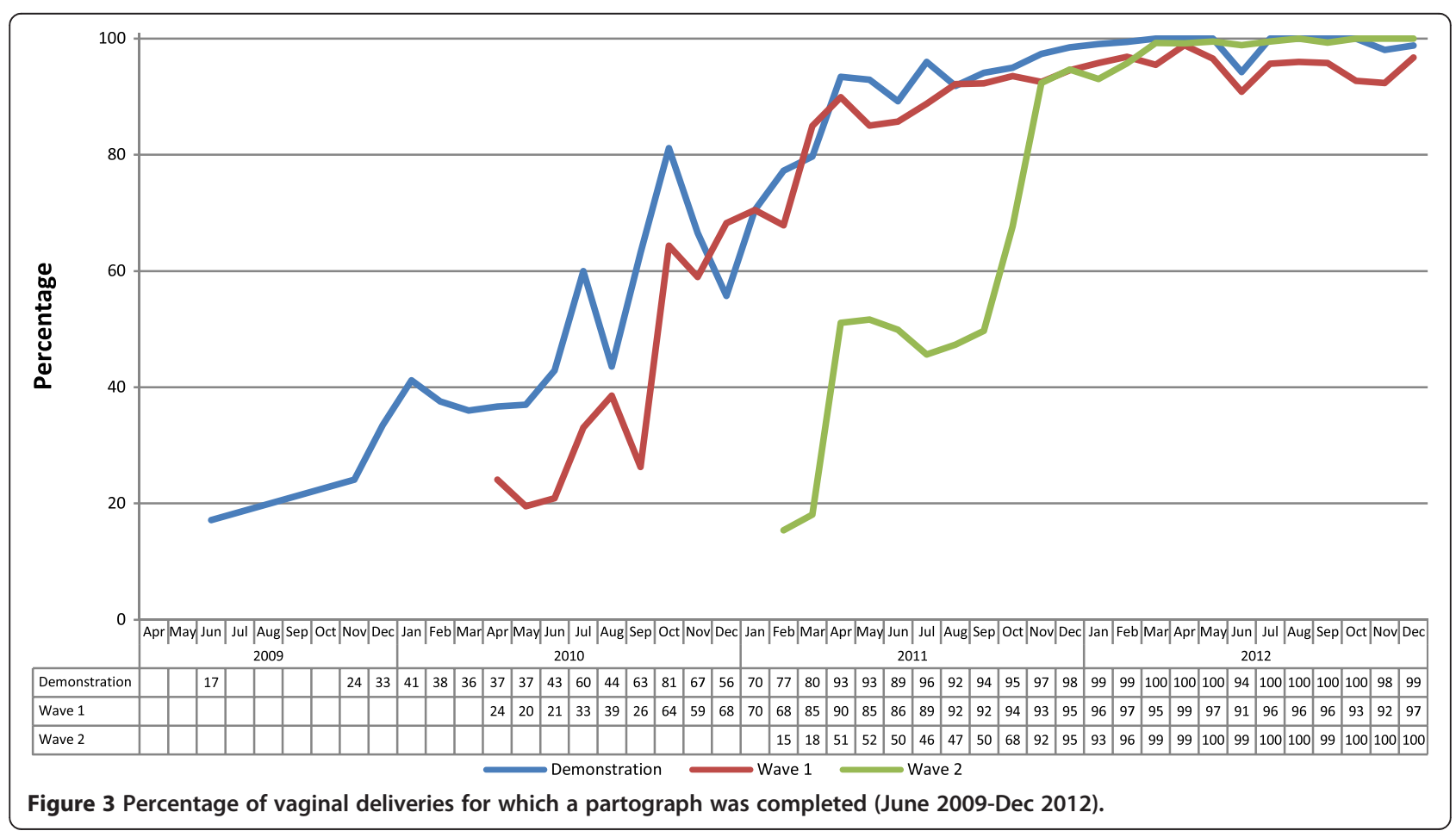



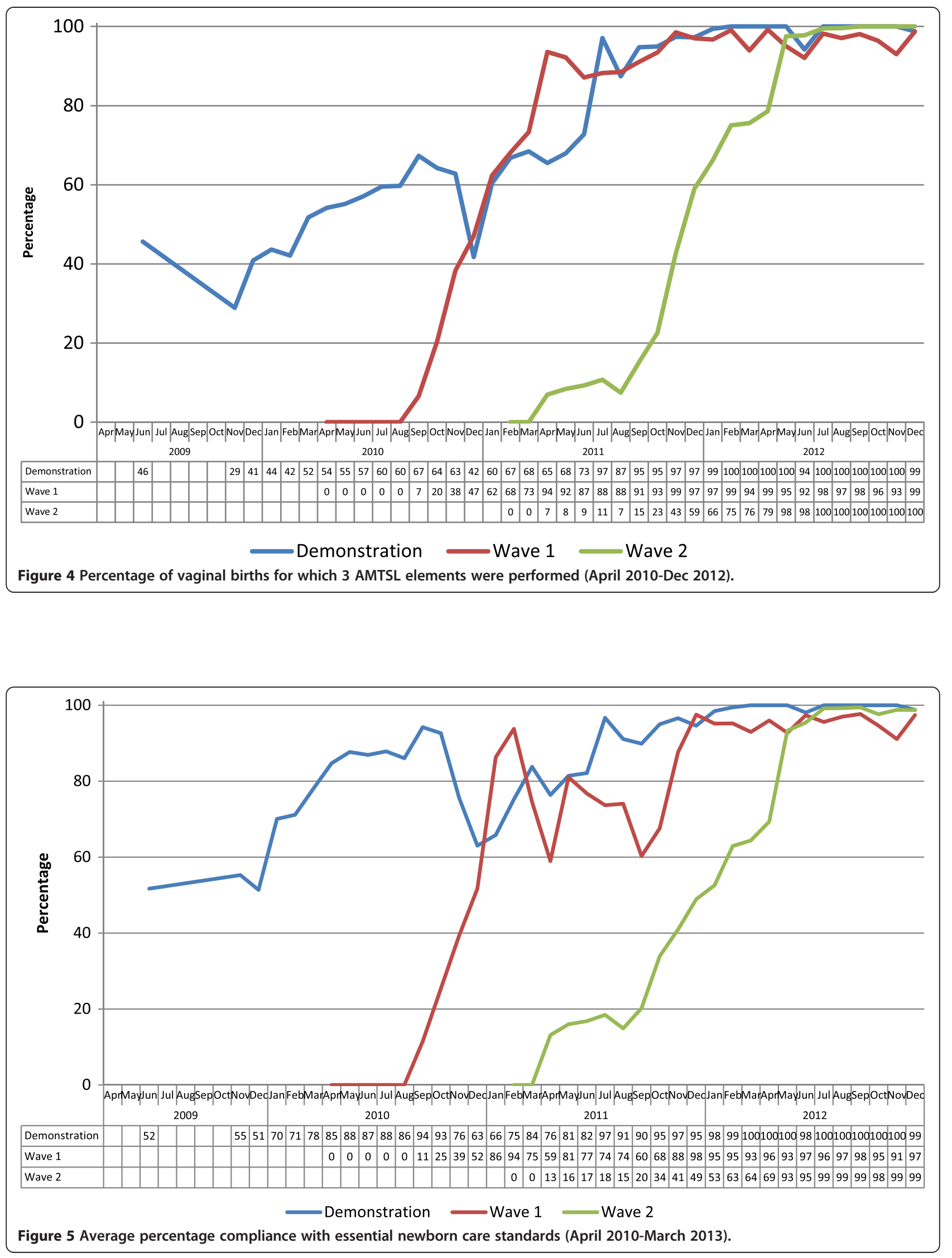


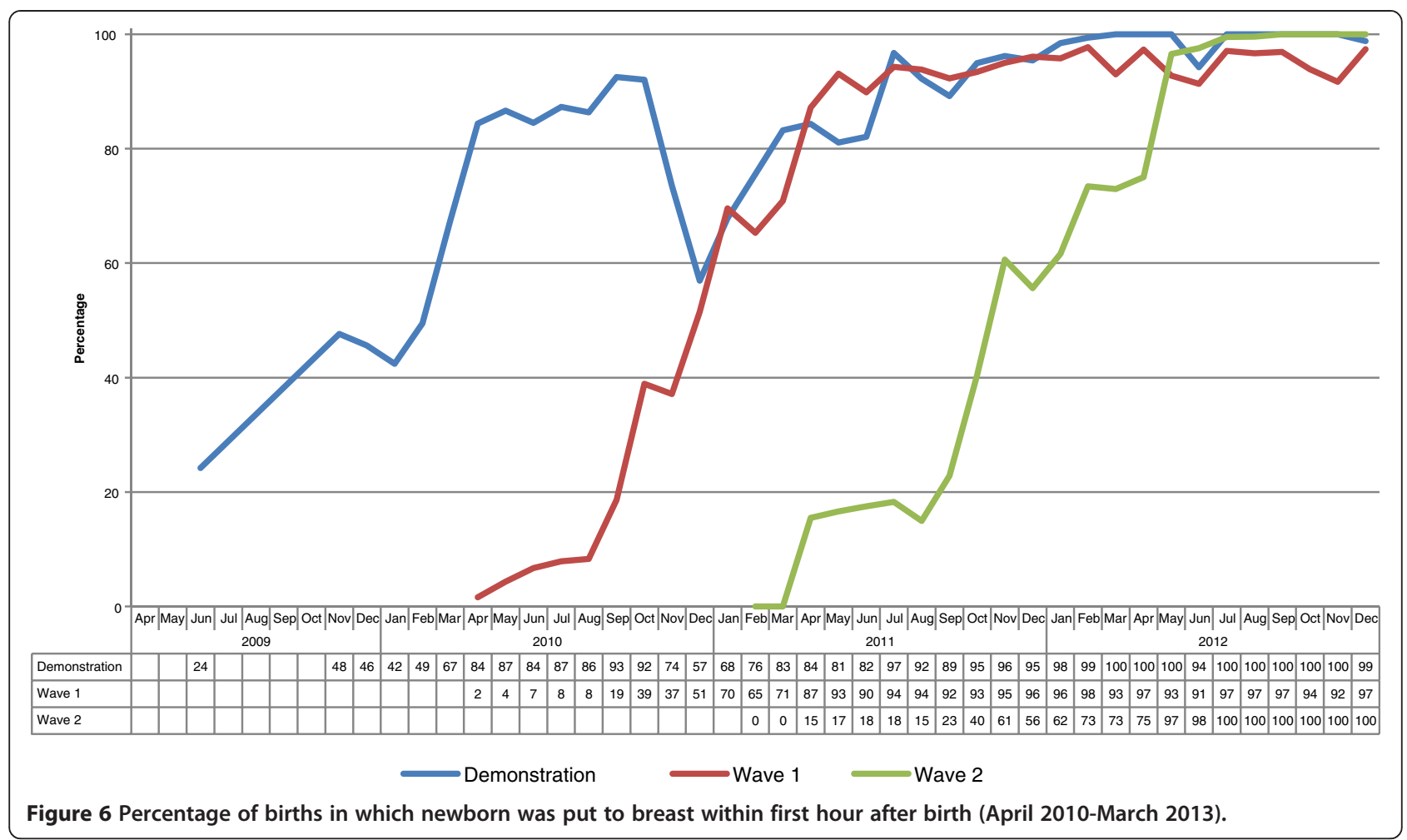

\section{Discussion and evaluation}

The last ten years have shown increased interest and recognition that human resource factors, such as the roles and responsibilities of frontline health workers, are central to improving health [8]. Data and studies are starting to emerge about the importance of engaging frontline health workers in improving performance [9]. Command and control approaches are losing their place within the health system. Communication within and between different parts of the system, which plays a

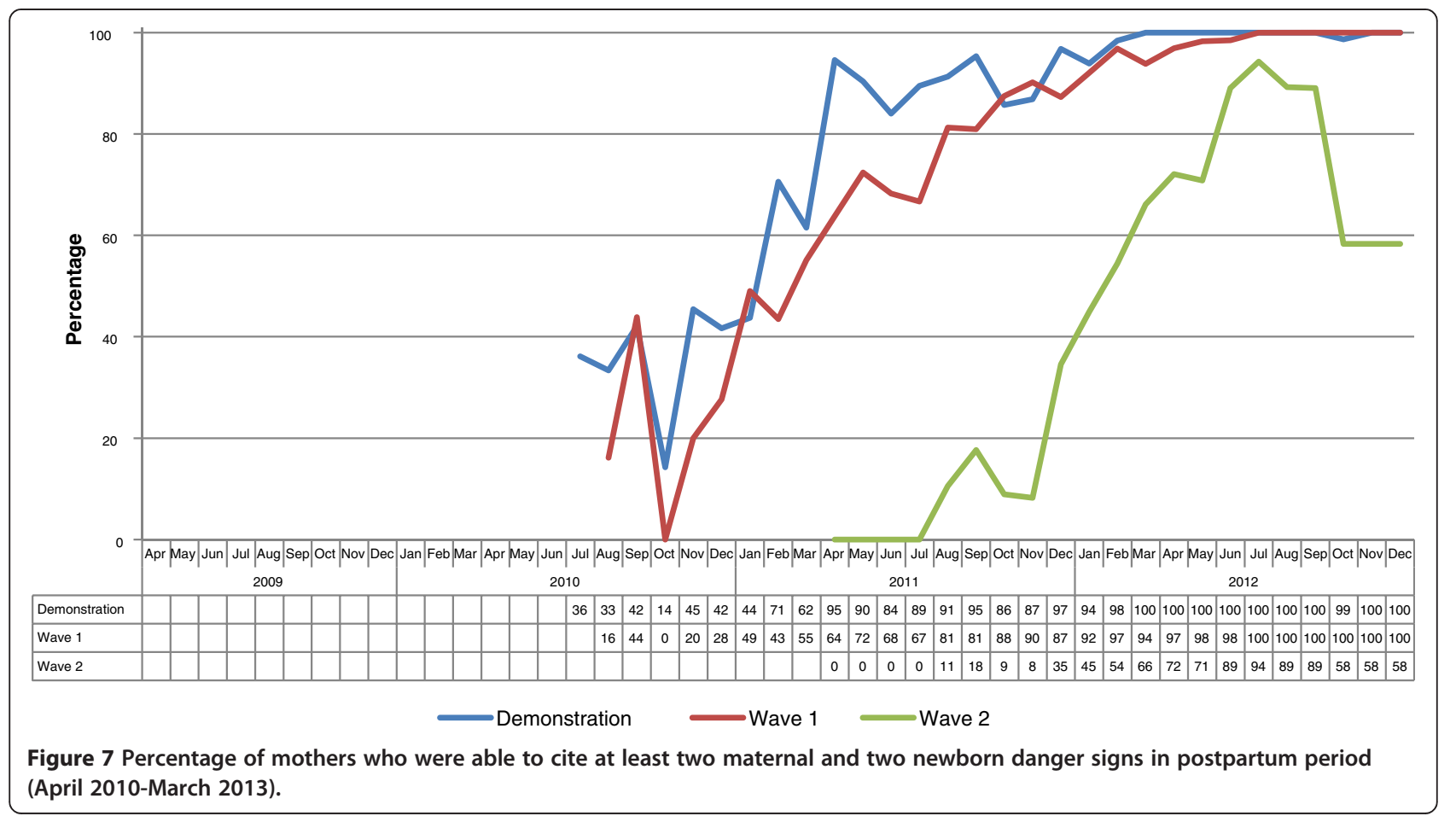


major role in improving quality of care, is receiving more attention.

Given that the tradition of identifying problems at the top level and sending solutions to frontline health workers has not proven to be effective in many developing countries, it is becoming increasingly important to define the roles and responsibilities and the engagement of frontline health workers.

While the Ministry of Health in Afghanistan made great strides to expand access to health services in the country in the last decade, gaps in the quality of care remained a challenge. The work conducted by the MoPH and $\mathrm{HCI}$ in Afghanistan over the last several years has shown that by engaging frontline health workers to work in teams, identify problems, and find local solutions for those problems, quality of care significantly improved. One example of local solutions this article reports was the limited use of the partograph during deliveries. There were numerous other local solutions used by the QITs in the country that are not reported in this article, such as use of prefillied syringes and labeling uterotonic to avoid mistaking it with local anesthetic on the tray for better adherence to AMTSL standards, establishing an internal review mechanism to oversee improvement work, improvement in data collection, and reporting after understanding the importance of measuring immediate outcomes such as postpartum hemorrhage. Local engagement created confidence and ownership among frontline providers to identify and introduce much better solutions that can improve quality of care. Witnessing their own improvements by the solutions they introduced gave health care workers trust and motivation to do more.

The data from the demonstration sites in Kunduz, Balkh, and Kabul showed that by engaging frontline workers, the quality of health services - and in turn the outcomes of care - can be significantly improved. Because of the results achieved in Kunduz and Balkh, the collaborative improvement work was expanded over time to seven more provinces. Results from these subsequent waves of improvement activities showed similar gains in health outcomes (Figures 3, 4, 5, 6 and 7). Quality improvement teams at the facility level, with a focal point at the provincial level connected to the $\mathrm{MoPH}$, will help to sustain these improvements in care quality.

In parallel to the facility-level collaboratives, improvement collaboratives were designed by $\mathrm{HCI}$ and Afghanistan-based experts to introduce high-impact, evidence-based interventions in maternal and newborn care at the community levels in Balkh and Kunduz provinces in order to link the different levels of care, from that provided in the home and community by traditional birth attendants and community health workers, all the way through to health posts, basic health centers, comprehensive health centers, and district and regional hospitals. The community-level collaborative was designed to improve the performance of individual community health workers in making antenatal and postnatal home visits and improve their counseling skills and strengthen community-to-facility linkages for increased uptake and coverage of skilled birth services, including prompt referral and management of maternal and newborn complications.

In addition, the results achieved on the ground in the provinces also led the MoPH to be interested in expanded HCI support for the ongoing development of the country's health care quality improvement strategy. To support the development of its strategy for quality in health care, in late 2009, the MoPH requested that $\mathrm{HCI}$, in collaboration with other partners, convene a panel of experts who could share relevant international experiences to help the MoPH make informed decisions in its journey to improve the quality of health services in Afghanistan.

To more effectively coordinate efforts for developing the strategy, the MoPH established the Unit for Improving Quality in Health Care (IQHC), and a Quality Task Force for the development of the Strategy for Quality in Health Care was organized, with participation of different departments of the $\mathrm{MoPH}$ and partner organizations, including HCI [10]. Following an almost year-long process of consultation and discussion, the National Strategy for Improving Quality in Health Care, consisting of a strategy implementation framework and five-year operational plan, was launched [4].

However, implementation of the facility-level collaborative did not come without its challenges. These included: high turnover of health facility staff in provinces; limited previous exposure to and understanding of basic improvement concepts among health facility staff, provincial public health officers, and frontline health workers; initial low motivation and commitment of health providers for the implementation of quality improvement efforts resulting from previous working relationships where they were not engaged and felt that they had no control over the problems their facilities faced; political insecurity in most of the provinces where HCI worked; shortage of essential supplies; lack of proper infrastructure for some health care facilities, including shortage of clean water, electricity, toilets and incinerators; and delay in salary payments for health care workers.

Most of the above-mentioned challenges remain beyond the control of any project or program within the health system. However, their consideration by the MoPH is still beneficial in planning for future quality improvement efforts. Motivation and commitment of health provinces can be improved if an appropriate reward and recognition mechanism (including a feedback system) is established. This type of reward and recognition mechanism was included in the Strategy for Quality in Health Care. In addition, the provincial QIT meetings discussed the challenges and recommended solutions for authorities. HCI 
also considered improving the frontline providers' inner motivation through integration of the psychology of change in learning sessions and coaching visits.

While the case study clearly shows a working strategy to enable frontline providers to enhance performance of health systems and shows signs of improvement in immediate outcomes, our results are limited in not having a rigorous sampling or design strategy. This article opens up the opportunity for future, more robust study designs using control groups or through enabling health information systems to collect data on the outcomes of interest, where logistically and ethically feasible.

\section{Conclusions}

Afghanistan's recent experience demonstrates that even under the most adverse of circumstances, quality of health care can be improved at the frontlines if there is coherent national leadership and direction coupled with effective technical support. The work described in Afghanistan has demonstrated how to achieve measurable improvements in actual patient care at the frontlines of service delivery while systematically building capacity at all levels of the health system through national leadership and policy making.

Improvement data from $2010-2013$ on about 100 health facilities in Afghanistan show that by engaging frontline workers in making changes to their care processes, the quality of health services can be significantly improved. This engagement gradually brings a cultural shift in understanding the roles and capabilities of frontline workers as well as their confidence and motivation. Health workers who were providing care as part of the system but working individually now work in teams, discuss problems, and introduce solutions by themselves. They feel more comfortable to meet with their supervisor and discuss issues that they were not comfortable discussing in the past. Data collected at the health facility level, which previously was mainly used for reporting to provincial level health departments, are now being used within the facility to plan the next course of action. Health workers who used to just implement the top-level decisions now take part in solving their local problems.

In late 2012, USAID requested that HCI phase out provincial level support in 2013 and prepare to close out its assistance to the MoPH. HCI closed all provincial offices by December 2012 and focused its support in the final year of assistance on the central level MoPH. Since the adoption of the National IQHC Strategy, the IQHC Unit assumed leadership for embedding the IQHC Strategy within all MoPH policies and strategies. The IQHC Unit also liaises with key MoPH directorates and departments, private sector agencies, United Nations agencies, and national and international NGOs for advocacy and institutionalization of a culture of quality and to enhancing capacity related to quality issues.
The ultimate goal of HCI's collaboration with the $\mathrm{MoPH}$ was to ensure that the IQHC Unit had the capacity and resources to oversee, coordinate, and maintain the mentioned approach at the national level and provide technical support and guidance to implementing NGOs on issues surrounding quality of health care in general and in harmony with other partners.

In 2013, a support structure to guide the QITs was established within the organigram of the MoPH. The IQHC Unit continues working with the QITs, focusing on the priorities the teams have selected for their health facilities. Implementation guidelines have been developed and approved for practice by the MoPH. Moreover, the $\mathrm{MoPH}$ has now earmarked a specific budget for improving quality in health care.

Afghanistan's experience suggests that the following factors contribute to measurable gains in health care quality at the facility level: 1) active engagement of frontline workers, 2) team work, 3) flexibility to focus on local priorities, 4) equipping frontline workers with basic improvement tools, and 5) improving communication between different parts of the system.

\section{Endnote}

${ }^{a}$ In Afghanistan, frontline health providers are those who are in direct contact with clients and patients. They can be health facility staff or community health workers. They are providing services to people in rural as well as urban areas.

\section{Abbreviations}

AMTSL: Active management of the third stage of labor; ANC: Antenatal care; ASSIST: USAID Applying Science to Strengthen and Improve Systems Project; BHC: Basic health center; BPHS: Basic Package of Health Services; CDC: Centers for Disease Control and Prevention; CHC: Comprehensive health center; DH: District hospital; EPHS: Essential Package of Hospital Services; HCl: USAID Health Care Improvement Project; HP: Health post; IQHC: Unit for Improving Healthcare; MoPH: Ministry of Public Health; NGO: Nongovernmental organization; PNC: Postnatal care; PPHD: Provincial Public Health Department; RH: Regional hospital; QI: Quality improvement; QITs: Quality improvement teams; UNICEF: United Nations International Children's Emergency Fund; USAID: United States Agency for International Development.

\section{Competing interests}

The authors declare that they have no competing interests.

\section{Authors' contributions}

MR led the USAID Health Care Improvement Project in Afghanistan and authored the manuscript. AJN is the Deputy Minister Policy and Planning Ministry of Public Health of Afghanistan who is leading the public health system in the country. He provided technical review of the document. SH is a Senior Improvement Advisor for the USAID ASSIST Project and contributed to drafting the manuscript and revising it critically for important intellectual content. AKH provided leadership to $\mathrm{HCl}$ activities at the provincial level initially only in Balkh province and later led $\mathrm{HCl}$ activities in all the provinces. He also edited and provided content to the manuscript. All authors read and approved the final manuscript.

\section{Acknowledgements}

The technical assistance in health care improvement provided to the Ministry of Public Health of the Islamic Republic of Afghanistan was supported by the American people through the United States Agency for International Development (USAID) and its Health Care Improvement Project $(\mathrm{HCl})$. 
$\mathrm{HCl}$ is managed by University Research Co., LLC (URC) under the terms of $\mathrm{HCl}$ Task Order 3, Contract Number GHN-1-03-07-00003-00. URC's subcontractors for HCl include EnCompass LLC, FHI 360, Health Research, Inc., Initiatives Inc., Institute for Healthcare Improvement, and Johns Hopkins University Center for Communication Programs.

This manuscript is part of the 'Filling the Void: Health systems in fragile and conflict affected states' thematic series.

\section{Author details}

'USAID Applying Science to Strengthen and Improve Systems (ASSIST) Project, University Research Co., LLC (URC), Alps Building, 1st Floor, 56, Janpath, New Delhi 110001, India. ${ }^{2}$ Ministry of Public Health, Kabul, Afghanistan. ${ }^{3}$ University Research Co., LLC (URC), 7200 Wisconsin Ave., Suite 600, Bethesda, MD 20814-4811, USA. ${ }^{4}$ USAID Health Care Improvement Project, Kabul, Afghanistan.

Received: 15 May 2014 Accepted: 15 September 2014

Published: 22 October 2014

\section{References}

1. Acerra JR, Iskyan K, Qureshi ZA, Sharma RK: Rebuilding the health care system in Afghanistan: an overview of primary care and emergency services. Int J Emerg Med 2009, 2(2):77-82.

2. Government of the Islamic Republic of Afghanistan: Strategic Plan for the Ministry of Public Health (2011-2015). http://www.gfmer.ch/SRH-Course-2012/ country-coordinators/pdf/Ministry-Public-Health-Strategic-Plan-2011-2015Afghanistan.pdf.

3. National Risk and Vulnerability Assessment. http://cso.gov.af/Content/ files/NRVA\%20REPORT\%20Introduction\%20Pages1.pdf.

4. Rahimzai M, Amiri M, Burhani NH, Leatherman S, Hiltebeitel S, Rahmanzai AJ: Afghanistan's national strategy for improving quality in health care. Int J Qual Health Care 2013, 25(3):270-276.

5. Franco $L M$, Marquez L: Effectiveness of collaborative improvement: evidence from 27 applications in 12 less developed and middle-income countries. BMJ Qual Saf 2011, 20:658-665.

6. University Research Co., LLC: The Improvement Collaborative: an approach to rapidly improve health care and scale up quality services. https://www.usaidassist.org/resources/improvement-collaborative-approachrapidly-improve-health-care-and-scale-quality-services.

7. University Research Co., LLC: Coaching as a tool to support quality improvement teams. https://www.usaidassist.org/sites/assist/files/ pisafcoachingmanual_english_2011_final.pdf.

8. Campbell J, Buchan J, Cometto G, David B, Dussault G, Fogstad H, Fronteira I, Lozano R, Nyonator F, Pablos-Mendez A, Quian EE, Starrs A, Tangcharoensathien $\checkmark$ : Human resources for health and universal health coverage: fostering equity and effective coverage. Bull World Health Organ 2013, 91:853-863.

9. The World Bank: Global Conference on Universal Health Coverage for Inclusive and Sustainable Growth: Lessons from 11 Country Case Studies: A Global Synthesis. http://documents.worldbank.org/curated/en/ 2013/11/18606533/global-conference-universal-health-coverage-inclusivesustainable-growth-global-synthesis-report.

10. Round Table Meeting on the National Improvement Strategy and Infrastructure for Improving Health Care in Afghanistan. https://www. usaidassist.org/resources/round-table-meeting-national-improvementstrategy-and-infrastructure-improvement-health.

doi:10.1186/1752-1505-8-21

Cite this article as: Rahimzai et al.: Engaging frontline health providers in improving the quality of health care using facility-based improvement collaboratives in Afghanistan: case study. Conflict and Health 2014 8:21.

\section{Submit your next manuscript to BioMed Central and take full advantage of:}

- Convenient online submission

- Thorough peer review

- No space constraints or color figure charges

- Immediate publication on acceptance

- Inclusion in PubMed, CAS, Scopus and Google Scholar

- Research which is freely available for redistribution

Submit your manuscript at www.biomedcentral.com/submit 